\title{
La Biblioteca Musical Económica y el Tesoro de Música Religiosa de Joaquín Espín y Guillén: dos ejemplos de iniciativa editorial privada decimonónica.
}

Joaquín Espín y Guillén fue compositor, crítico y musicógrafo, y desarrolló su actividad a lo largo del s. XIX. Contribuyó al desarrollo de la ópera española desde diferentes ámbitos: compuso Padilla o el asedio de Medina (que fue vista por muchos como la primera ópera española) y un buen número de canciones españolas, y organizó veladas musicales en su propio domicilio que generaron un espacio donde los diletantti podían practicar el canto en castellano y en el cual los compositores testaban la idoneidad de sus obras vocales escritas en la lengua materna. Durante toda su vida ejerció en distintos periódicos como crítico musical, realizando una defensa constante del drama lírico nacional. Una de sus aportaciones más valiosas al desarrollo de la música española fue la fundación del primer periódico musical de nuestro país, La Iberia Musical, en 1842. Su importancia radica no sólo en la reivindicación de la protección de la música española que Espín y los demás redactores llevaron a cabo desde sus páginas -lo cual cristalizó, principalmente, en una férrea defensa del drama lírico nacional frente a la ópera italiana- sino también en los suplementos musicales que con ella se repartieron, donde dio a conocer a sus suscriptores las obras vocales de compositores españoles coetáneos, además de parte del repertorio lírico italiano en boga.

Aunque tanto la Biblioteca Musical Económica como el Tesoro de Música Religiosa fueron consideradas en su época como periódicos musicales ${ }^{1}$, en realidad se trataba en ambos casos de colecciones o antologías musicales. En este sentido, los suplementos musicales repartidos con La Iberia Musical y Literaria entre 1842 y 1846 pueden considerarse como un antecedente, aunque con mayor importancia en el caso de la Biblioteca, cuyas partituras se clasifican en función de los mismos géneros que los estipulados para los suplementos: canto y piano, piano solo.

La Biblioteca Musical Económica se inició en septiembre de $1852^{2}$, en un momento en que eran habituales empresas editoriales de características similares aunque relativas a otras ciencias, una práctica frecuente ya en la década anterior. En este sentido, podemos citar la

\footnotetext{
${ }^{1}$ Véase, por ejemplo: "Noticias generales”, La Época, 17-XI-1852, n. 1424.

2 "Biblioteca Musical Económica", El Correo de los Teatros, 22-VIII-1852, n. 39.
} 
Biblioteca de Autores Españoles, vinculada con la Literatura -que comenzó a publicarse en 1846 bajo la dirección de Carlos Anibau ${ }^{3}$ - o la Biblioteca Económica de Medicina y Cirugía, que se encontraba ya a la venta en enero de $1851^{4}$, bajo la dirección de Jose María Velasco y Manuel Álvarez Chamorro. Pero existían también algunos ejemplos de iniciativas editoriales musicales publicadas bajo el mismo tipo de denominación, como la Biblioteca Lírica Antigua y Moderna, dirigida por Antonio Jose Cappa ${ }^{5}$. Esta colección de partituras comenzó a publicarse en septiembre de $1851^{6}$, y estaba constituida por 10 secciones que abarcarían la "música instrumental, música para piano solo, música vocal e instrumental para salón, música de baile para piano y para piano y orquesta y puramente instrumental, y música nacional"7 . Sin embargo, en el caso del Tesoro de Música Religiosa -que comenzó a publicarse a principios enero $1853^{8}$ - no existía un contexto editorial tan activo, probablemente porque los aficionados musicales no demandaban tanto la música sacra como la lírica. No obstante, esta colección de música religiosa se inició cuando la monumental obra de Hilarión Eslava, la Lira Sacro-Hispana, llevaba ya editándose casi un año (aproximadamente, desde mayo de 1852) ${ }^{9}$.

\section{Biblioteca Musical Económica}

\section{Características generales}

La Biblioteca Musical Económica fue concebida por Espín como una colección de partituras "al alcance de todas las inteligencias y de todas las edades"10 y estaría constituida por "la música más elegida de los compositores célebres [...] sirviendo de recreo, instrucción y utilidad a los profesores, colegios y diletantti, surtiéndoles de cuanta nueva

\footnotetext{
3 "Variedades", El Clamor Público, 15-I-1846, n. 594.

4 “Índice”, Boletín de Medicina, Cirugía y Farmacia, 5-I-1851, n. 1.

5 Antonio José Cappa Maqueda fue compositor y director; se formó en el Conservatorio de Música de Nápoles, donde estudió piano y composición; se embarcó en varios proyectos como editor musical, entre ellos, la mencionada colección. Véase MARTin Tenllado, Gonzalo. “Cappa Maqueda, Antonio José”. En: Diccionario de la Música Española e Hispanoamericana. Madrid: SGAE, 1999. Vol. III, pp. 140-141.

6 "Variedades", El Clamor Público, 14-VIII-1851, n. 2177.

7 "Biblioteca lírica antigua y moderna”, El Clamor Público, 16-VIII-1851, n. 2179.

8 “Sección de anuncios. Tesoro de música religiosa”, La España, 12-IV-1853, n. 1543.

9 “Publicación de anuncios", La Esperanza, 21-VI-1853, n. 2655.

10 “Anuncios. Biblioteca Musical Económica”, El Correo de los Teatros, 22-VIII-1852, n. 39.
} 
música puedan apetecer por un precio altamente económico" ${ }^{11}$. A pesar de lo indicado en el anuncio, la colección de partituras que se presentó al público no tocó todos los géneros, sino que se centró en la canción y en la pequeña forma de la música de cámara para piano. Sin embargo, Espín preveía que las dos secciones iniciales -canto y piano, y piano solopodrían ser ampliadas en función del éxito de la Biblioteca; se hubieran incluido entonces la reducción de óperas completas para canto y piano, y también la reducción para piano solo, aunque no llegó a concretarse finalmente.

En un inicio, se repartían dos entregas de cada sección al mes, que salían en semanas alternas; a partir de marzo de 1853 se incluyó la opción de entregar más de dos por sección; cada entrega constaba de "cuatro láminas grandes de música impresa en excelente papel y grabado" ${ }^{2}$. Los suscriptores podían optar por abonarse a ambas secciones o solamente a una, pero debían pagar un depósito de 4 reales que se perdía si el suscriptor no completaba el tomo. El precio de cada entrega era de 2 reales, medio real más para la suscripción en provincias. Podían adquirirse las entregas sueltas, pero el coste pasaba a ser el doble. Si comparamos estos precios con los estipulados por Jose Cappa para su Biblioteca Lírica Antigua y Moderna, observamos que la oferta de Joaquín Espín era algo más económica. En el primer caso, salían dos entregas al mes de "diez páginas [...] de correcta, clara y compacta impresión, pliego de marca francés en hermoso papel"13, a 8 reales cada entrega y 12 reales en provincias. Dicho de otro modo, Cappa ofrecía 20 páginas de música por 16 reales (sin depósito previo), y Espín presentaba 16 láminas de música por 8 reales, ambos precios estipulados para la capital. La suscripción a la Biblioteca Musical Económica se realizaba en todos los almacenes de música, en las librerías de Monier, Bonat y Baillieri (distribuidoras de la colección musical dirigida por Cappa), en el Gabinete Literario de Mellado (cuyo establecimiento tipográfico editó durante algunos meses La Iberia Musical en 1842) y el domicilio del propio Espín, situado desde 1845 en la calle de Luna, nº 26.

De los dos tomos de los que constó esta colección, el primero ya estaba terminado en octubre de $1853^{14}$, e incluía 21 partituras para canto y piano, y 19 partituras para piano solo.

\footnotetext{
11 “Anuncios. Biblioteca Musical Económica”, La España, 3-III-1853, n. 1511.

12 “Anuncios. Biblioteca Musical Económica”, El Correo de los Teatros, 22-VIII-1852, n. 39.

13 "Biblioteca lírica antigua y moderna”, El Clamor Público, 16-VIII-1851, n. 2179.

14 “Biblioteca Musical Económica”, La España, 19-X-1852, n. 1702.
} 
Fueron repartidas mediante 25 entregas en ambos casos. En abril de 1854 la primera y segunda parte -ambos tomos- ya estaban completas ${ }^{15}$. Únicamente hemos podido constatar de manera íntegra la totalidad de las entregas del Tomo I de ambas secciones.

\section{Repertorio}

\section{CUADRO I}

Relación de partituras correspondientes a la sección Canto y Piano, Tomo I. Biblioteca Musical Económica

\begin{tabular}{|c|c|c|c|c|}
\hline TÍTULO & SUBTÍTULO & VOZ / VOCES & $\begin{array}{c}\text { AUTOR } \\
\text { LITERARIO }\end{array}$ & COMPOSITOR \\
\hline La rosa & Melodía italiana & Mezzosoprano & T. Solera & Espín \\
\hline La noche & $\begin{array}{l}\text { Duettino } \\
\text { nocturno }\end{array}$ & $\begin{array}{c}\text { Tiple } \\
\text { Contralto }\end{array}$ & & Espín \\
\hline La pastorella & $\begin{array}{l}\text { Canzoneta } \\
\text { italiana }\end{array}$ & Soprano & $\begin{array}{l}\text { N di Santo- } \\
\text { Magno }\end{array}$ & Rossini \\
\hline Recuérdalo & $\begin{array}{l}\text { Romanza } \\
\text { española }\end{array}$ & & P. Madrazo & Espín \\
\hline Red de amores & $\begin{array}{l}\text { Canción } \\
\text { española }\end{array}$ & & $\begin{array}{l}\text { Romero } \\
\text { Larrañaga }\end{array}$ & Espín \\
\hline Beltá crudele & Melodía & Soprano & $\begin{array}{l}\text { N. di Santo- } \\
\text { Magno }\end{array}$ & Rossini \\
\hline L'invito & Romanza & Contralto & & Espín \\
\hline $\begin{array}{l}\text { Cavatina del final del Acto } \\
\text { IV de Roberto il diabolo }\end{array}$ & & & & Meyerbeer \\
\hline Il resentimento & Romanza & & & Rossini \\
\hline La lontananza & Romanza & Soprano & & Espín \\
\hline La jamona & $\begin{array}{l}\text { Canción } \\
\text { española }\end{array}$ & & & Espín \\
\hline $\begin{array}{l}\text { Lamento por la muerte de } \\
\text { Bellini }\end{array}$ & & & & Donizetti \\
\hline La serenata & Romanza & Tenor / soprano & & Espín \\
\hline El sueño & $\begin{array}{l}\text { Romanza } \\
\text { española }\end{array}$ & & P. Madrazo & Espín \\
\hline La pesca & Duettino & $\begin{array}{l}\text { Soprano } \\
\text { Contralto }\end{array}$ & Metastasio & Rossini \\
\hline El barquero del Tajo & Barcarola & $\begin{array}{l}\text { Contralto / } \\
\text { Barítono }\end{array}$ & P. Madrazo & Espín \\
\hline La serenata & $\begin{array}{l}\text { Duettino } \\
\text { nocturno }\end{array}$ & $\begin{array}{l}\text { Soprano } \\
\text { Contralto }\end{array}$ & Pepoli & Rossini \\
\hline $\begin{array}{l}\text { Romanza de Beatrice di } \\
\text { Tenda }\end{array}$ & & $\begin{array}{l}\text { Barítono / } \\
\text { contralto }\end{array}$ & & Bellini \\
\hline Aria de la ópera Rigoletto & & Tiple & & Verdi \\
\hline Dueto de Don Giovanni & & $\begin{array}{c}\text { Soprano } \\
\text { Bajo }\end{array}$ & & Mozart \\
\hline $\begin{array}{l}\text { Romanza de Il dui ilustri } \\
\text { rivali }\end{array}$ & & & & Mercadante \\
\hline
\end{tabular}

\footnotetext{
${ }^{15}$ Velaz de Medrano, Eduardo. "Revista musical”, La España, 6-IV-1854, n. 1846.
} 
Como se observa en el Cuadro I, del total de obras de la sección de canto y piano del Tomo I, 10 obras pertenecen a Espín, 5 piezas son obra de Rossini y las restantes pertenecen a algunos de los compositores más apreciados en el Madrid decimonónico, como Donizetti, Bellini, Verdi o Meyerbeer. Espín agrupó en esta sección fragmentos de óperas muy conocidas del primer y segundo romanticismo italiano, como la romanza perteneciente a la ópera Beatrice di Tenda, de Bellini, o el aria de Gilda, "Caro nome", del Acto I de Rigoletto, de Verdi, que fue entregada a los suscriptores antes de que se estrenase la ópera en el Teatro Real de Madrid, el 16 de octubre de $1853^{16}$. También incluyó la cavatina final de Isabelle, "Robert, toi que j'aime", de Roberto le diable, de Meyerbeer (que fue interpretada en el Teatro Real en $1853^{17}$ ) pero en su traducción al italiano, emulando la práctica habitual en Madrid por la cual se traducían a este idioma aquellas óperas no italianas. Aunque el Don Giovanni de Mozart no fue en su estreno en Madrid bien acogida por el público que ya entonces había sucumbido al denominado "furor filarmónico" y a la ópera italiana del primer romanticismo, y a pesar de que en años posteriores la obra lírica mozartiana no tuvo una presencia tan acusada en los teatros madrileños en comparación con la ópera italiana o la Grand opéra francesa, Espín incluyó en esta colección de música el dúo de Giovanni y Zerlina "Là ci darem la mano" (Acto I) de Don Giovanni.

Además de estos fragmentos operísticos, las canciones italianas y españolas formaban el grueso de la sección de canto y piano. La gran mayoría son de obra de Espín, que aprovechó esta empresa editorial como un medio para publicitarse como compositor, al igual que anteriormente lo había hecho en los suplementos que repartía con La Iberia Musical y Literaria. Éste fue el caso de su canción La Aldeana (poesía de Bretón de los Herreros), cantada por la prima donna Adelaida Perelli en el Teatro de la Cruz ${ }^{18}$, y de $L a$ Caprichosa (poesía de Agustín Príncipe), cantada por Rosalía Gariboldi en el Teatro del Circo, en $1843^{19}$. Del mismo modo, Espín introdujo en esta colección musical algunas de aquellas canciones que habían disfrutado anteriormente de una buena acogida entre los aficionados madrileños, como la canción Red de amores (poesía de Romero Larrañaga), no

\footnotetext{
16 “Crónica de teatros. Rigoletto”, El Clamor Público, 14-X-1853, n. 2837.

17 "Noticias generales", La Época, 4-IV-1853, n. 1244.

18 “Crítica musical. L'elixir de amore”, La Iberia Musical y Literaria, 20-II-1842, n. 8, p. 30.

19 “Crónica Nacional”, La Iberia Musical y Literaria, 4-VI-1843, n. 23, p. 183.
} 
obstante, la inclusión de sus canciones españolas -La jamona, Recuérdalo, etc.- es mucho menor en comparación con la presencia de canciones italianas. En relación a éstas, las obras de Espín comparten protagonismo con las de Rossini; de este autor, dos de ellas pertenecen al álbum Les soirèes musicales, editado en París en la década de los años treinta (La pesca y La serenata), mientras que las otras tres (La pastorella, Il resentimento y Beltà crudele) no pertenecen a un mismo álbum y fueron escritas en diferentes años.

\section{CUADRO II}

\section{Relación de partituras correspondientes a la sección Piano solo, Tomo I} Biblioteca Musical Económica.

\begin{tabular}{|c|c|c|c|}
\hline TÍTULO & SUBTÍTULO & $\begin{array}{c}\text { AUTOR } \\
\text { ÓPERA /ZARZUELA }\end{array}$ & COMPOSITOR \\
\hline $\begin{array}{l}\text { Vals y pastoral de } I l \\
\text { profeta }\end{array}$ & & Meyerbeer & Desconocido \\
\hline La Granja & Polka-mazurka & & Espin \\
\hline Primer nocturno & & & Dölher \\
\hline $\begin{array}{l}\text { Recuerdos de la } \\
\text { ópera Don Sebastián }\end{array}$ & & Donizetti & Desconocido \\
\hline Aranjuez & Polka & & Espín \\
\hline $\begin{array}{l}\text { Segundo nocturno, } \\
\text { op. } 25\end{array}$ & & & Dölher \\
\hline $\begin{array}{l}\text { Recuerdos de Luisa } \\
\text { Miller }\end{array}$ & & Verdi & Desconocido \\
\hline El Escorial & Polka-mazurka & & Espín \\
\hline $\begin{array}{l}\text { [Buenas noches } \\
\text { señor] Don Simón }\end{array}$ & Vals & Oudrid & Desconocido \\
\hline $\begin{array}{l}\text { Recuerdos de la } \\
\text { ópera I martiri }\end{array}$ & & Donizetti & Desconocido \\
\hline El judio errante & Tanda de valses & & Musard \\
\hline Nocturno de salón & & & Dölher \\
\hline $\begin{array}{l}\text { Zerlina o el } \\
\text { canastillo de } \\
\text { naranjas }\end{array}$ & Schothis & Auber & Desconocido \\
\hline El pensamiento & Polka-mazurka & & Espín Pérez \\
\hline $\begin{array}{l}\text { Fantasía sobre los } \\
\text { mejores motivos de } \\
\text { Il Puritani }\end{array}$ & & Bellini & Döhler \\
\hline $\begin{array}{l}\text { Tanda de rigodones } \\
\text { de El profeta }\end{array}$ & & Meyerber & Desconocido \\
\hline $\begin{array}{l}\text { El carnaval de } \\
\text { Venecia }\end{array}$ & Variaciones brillantes & & Hertz \\
\hline Simpatía & Vals & & Espín \\
\hline $\begin{array}{l}\text { La pequeña } \\
\text { campana del } \\
\text { cementerio }\end{array}$ & Nocturno & Carvalho (João de Sousa) & \\
\hline
\end{tabular}


En el Cuadro II podemos observar las partituras que conformaron el Tomo I de la sección dedicada al piano, en la que Espín y Dölher son los dos compositores más recurrentes, incluyendo 4 obras de cada uno de ellos, junto con una discreta presencia de obras Auber, Hertz e incluso, de Joaquín Espín Pérez de Colbrand, su hijo. Las pequeñas formas de salón (vals, polka, etc.) son las predominantes aquí, donde quizás se pueda destacar la presencia de los tres nocturnos del pianista Dölher, posiblemente incluidos en esta sección debido a la inclinación que Espín tenía por la escuela pianística iniciada por Czerny (maestro de Dölher) ${ }^{20}$; posiblemente, el mismo criterio haya sido aplicado para la introducción de unas variaciones de Hertz en este apartado. En relación a las obras del propio Espín, los títulos de algunas de sus piezas para piano, como la denominada Aranjuez o la titulada El Escorial, nos lleva a pensar en una posible inspiración musical de tipo españolista aunque, dado que las partituras de estas obras no se han conservado, no podemos constatarlo. Al lado de la pequeña forma de salón proliferan también las piezas inspiradas en óperas conocidas y apreciadas en la época, ya sean fantasías, como la realizada por Dölher sobre la ópera Il puritani, de Bellini, o formas de danza, como el vals y pastoral inspirado en Le prophéte, de Meyerbeer, o el schotis basado en la ópera Zerline de Auber. Desafortunadamente, desconocemos los autores de estas composiciones. Salvo la fantasía basada en la ópera de Bellini y la obra inspirada en la ópera Luisa Miller de Verdi, debemos destacar cómo las demás piezas para piano están inspiradas en óperas francesas del Romanticismo, con preferencia por la Grand Opéra.

\section{Edición, difusión y conservación de la Biblioteca Musical Económica}

Analizar las características de la edición de las obras que conformaban esta colección es un tema complejo, puesto que la mayor parte se halla perdida en la actualidad -como L'invito, El sueño, y La jamona, todas ellas obra de Espín- permaneciendo algunos ejemplares de sus canciones mediante ediciones anteriores o posteriores: Red de amores es conservada mediante su edición previa como suplemento de La Ópera; La lontananza y La serenata, han llegado hasta la actualidad gracias a una edición posterior realizada por Antonio Romero, conservándose un ejemplar de ambas en la Biblioteca Nacional de España, o podemos citar el ejemplo de El trovador del Tajo, que presenta una impresión posterior (ca.

\footnotetext{
${ }^{20}$ Sabemos que Espín se trasladó a Burdeos a estudiar con Hoffman, quien entronca con esta tradición pianística.
} 
$1864^{21}$ ) que las realizadas para la Biblioteca Musical Económica, anónima y más lujosa, lo que se trasluce en la introducción de un grabado que ejemplifica su título. Espín se la dedicó al rey portugués Fernando Augusto Sajonia Coburgo-Gotha (padre del rey Pedro V de Portugal), lo cual explicaría esta vistosa edición, que se conserva en la Biblioteca musicale governativa del Conservatorio di musica de Santa Cecilia (Roma).

Como ejemplo de las escasas partituras conservadas en su edición para la Biblioteca Musical Económica, nos centraremos en la canción italiana La Rosa (poesía de Temístocle Solera), música del director de esta antología. Está dedicada a la señora Dolores de Ardoy, una de las aficionadas que participaban en el Círculo Filarmónico fundado por Espín, en el que solían interpretarse las piezas publicadas en esta Biblioteca, al igual que en la década de los cuarenta sucedió con los suplementos musicales de La Iberia Musical y Literaria, que frecuentemente formaron parte del programa de los conciertos organizados por la redacción de este periódico.

La Rosa fue grabada en la Litografía de Antonio Hermoso, cuando su almacén de música estaba situado en la calle Mayor, es decir, entre 1826 y 1847. Por tanto, cabe suponer que la canción ya debía estar impresa antes de que Espín iniciase la Biblioteca, lo cual parece tener relación con la ausencia en esta impresión de los términos "Propiedad" y "Depositado", habituales a partir de la Ley de la Propiedad Intelectual de 1847. En la parte inferior izquierda de la primera lámina de la partitura se indica de manera desglosada "Biblioteca Musical Económica"; en la parte inferior derecha, aparece indicado Cuaderno 1 y 2 , y el número 1 , que se repite en cada una de las láminas de la canción y que alude al número de entrega. Estas referencias podemos considerarlas como si se tratase del número de plancha, aunque no presente el formato habitual. Sería posible que la ausencia de un número de plancha como tal se debiese a un volumen de producción poco elevado, pero lo cierto es que La Rosa tuvo tanto éxito que en octubre de 1852 hubo de prepararse una segunda tirada ${ }^{22}$. De hecho, en la prensa se llega a comentar que su éxito está vinculado a la

\footnotetext{
${ }^{21}$ Según consta en las fuentes hemerográficas, Espín compuso esta pieza dedicada al regente de Portugal, quien le otorgó la condecoración de la Orden del Cristo. Véase: “Gacetillas”, La Discusión, 15-I-1864, n. 2474.

22 “Biblioteca Musical del maestro Espín y Guillén”, El Correo de los Teatros, 10-X-1852, n. 46.
} 
adopción del estilo rossiniano por parte de Espín ${ }^{23}$. En cuanto al diseño de la portada, hemos de señalar que se corresponde con la tipología habitual durante la primera mitad del siglo XIX, siguiendo la tradición inglesa de grabados tipográficos sin apenas motivos figurativos, de gran sencillez, seguramente debido al interés de ofrecer una edición de calidad sin alcanzar unos costes excesivamente elevados en su producción. La caligrafía es vertical, también de influencia inglesa.

Sin embargo, no parece que éstas fueran las características habituales en las impresiones realizadas a partir de 1850. La canción Red de amores (poesía de Romero Larrañaga) fue compuesta por Espín para Erminia Frezzolini, prima donna del Teatro Real, quien la interpretó con éxito en su beneficio el 8 de abril de $1851^{24}$. A finales de mes, fue repartida como suplemento del periódico La Ópera ${ }^{25}$, y es esta edición y no la de la Biblioteca Musical Económica la que se conserva actualmente. La portada fue realizada en la Calcografía de Lodre y la partitura por el calcógrafo José Carrafa, quien realizó numerosos trabajos para la editorial familiar de Bernabé Carrafa, que se encargaba de editar el periódico al que esta canción pertenecía en calidad de entrega musical ${ }^{26}$.

En comparación con la La Rosa se aprecian en Red de amores algunas diferencias en las características de impresión que queremos comentar como ejemplo del cambio en las pautas de impresión entre la primera y segunda mitad del s. XIX. En la portada de La Rosa ya no aparecen los grabados de influencia inglesa (que tan sólo se aprecia en la tipografía) y se presenta vacía de adornos y figuraciones. Sin embargo y a pesar de encontrarse ya vigente la Ley de la Propiedad Intelectual de 1847, no consta que la obra fuese registrada y tampoco presenta número de plancha como tal; solamente se indica en la parte inferior izquierda el número del suplemento con el que se corresponde.

\footnotetext{
23 “Álbum”, El Correo de los Teatros, 7-X-1852, n. 45.

24 “Teatro Real. Beneficio de la Frezzolini”, La Ópera, 13-III-1851), n. 15, p. 2.

25 “Álbum”, La Ópera, 29-IV-1851, n. 16, p. 7.

${ }^{26}$ Lodre ya había trabajado con Espín durante los años que éste consiguió mantener en circulación La Iberia Musical y Literaria, encargándose de la parte musical desde el enero de 1842, tomando el relevo Bernabé Carrafa a partir de marzo de 1843.
} 
Desconocemos si Espín pudo reaprovechar las planchas para volver a imprimir esta obra al incluirla en la Biblioteca Musical Económica un año después de su estreno ${ }^{27}$, aunque no parece inverosímil, dado que incluso el precio estipulado para la obra coincide en su versión como suplemento de La Ópera y como parte de esta colección musical. Sorprende, sin embargo, que no se haya conservado algún ejemplar más de esta canción ya que en 1854 volvía a anunciarse su venta al público de manera independiente de la Biblioteca de Espín $^{28}$.

Como ejemplo de la probable reutilización de las planchas musicales de algunas de las obras de Espín ya impresas al inicio de esta antología, podemos utilizar las similitudes que encontramos entre la impresión de la polka-mazurka La Granja -perteneciente a la sección de piano solo- y la polka Angelita, que se repartió -al igual que Red de amores- como suplemento de La Ópera. El tipo de letra es en ambas vertical, de influencia inglesa, y la única diferencia reside en la inclusión de las siglas que vinculan La Granja con la antología de Espín (B. M. E.) y el precio de la entrega (4 reales), sin hacer mención a un posible número de plancha ni al autor del grabado o de la casa calcográfica o litográfica donde se realizó.

En cuanto a las vías de difusión de la Biblioteca, hemos localizado dos: la recomendación a través de amigos y conocidos y la publicidad en la prensa de la época. En cuanto a la primera, tenemos constancia de una carta escrita por Espín a Barbieri, en la que le envía una muestra de su Biblioteca y le solicita que "la recomiendes a toda la clientela y amigos" ${ }^{29}$. En relación con la segunda opción, se ha localizado una reseña de El Correo Sevillano que publicó El Correo de los Teatros, por la cual hemos podido saber del anuncio de la Biblioteca Musical Económica también en provincias:

La que va a aparecer en la corte bajo la dirección de un distinguido maestro, Don Joaquín Espín y Guillén, muy apreciado por sus esfuerzos en pro de la ópera nacional, y por las muchas obras que se han debido a su incansable pluma, está llamada a prestar un inmenso servicio al arte, porque por ella todos los aficionados desde el más alto personaje hasta el que habita en las pequeñas poblaciones hallan medio de solaz y de instrucción [...] Creemos que la biblioteca que

\footnotetext{
27 "Sección de anuncios. Biblioteca Musical Económica”, La España, 24-II-1853, n. 1505.

28 "Sección de anuncios. Red de amores", La España, 31-III-1853, n. 1841.

${ }^{29}$ EsPín Y GuILlÉN, Joaquín. Carta dirigida a Francisco Asenjo Barbieri, 17-IX-1852. Mss 14028-71.

Biblioteca Nacional de España.
} 
hoy nos ocupa, y en la que preside un gran pensamiento, va a ser recibida con interés por el mundo filarmónico. El nombre del maestro que figura a su frente basta para que merezca la aprobación de los que por afición o por necesidad cultivan el arte ${ }^{30}$.

Pero quizás la vía más importante para su difusión fueron los anuncios insertados en los diferentes periódicos de la capital; en el caso de El Clamor Público, La Esperanza, El Heraldo y El Correo de los Teatros, los anuncios son puntuales, incluidos tan sólo para notificar la próxima entrega o la ya realizada. No guardan ningún tipo de periodicidad y no parece que exista una razón por la cual se incluyan en estos periódicos en concreto. Sin embargo, en La España encontramos una constante referencia a la colección de partituras dirigida por Espín: es frecuente localizar entre 2 y 4 anuncios por mes, indicando las entregas realizadas y las que quedan por llevar a cabo. Dicho periódico era distribuido también por provincias, lo que aumenta su importancia en cuanto a la difusión de esta antología. Del mismo modo, creemos que el mayor volumen de anuncios de la Biblioteca Musical Económica que presenta La España está relacionado con su orientación política, pues Espín estaba más ligado a la tendencia moderada (que también abrazaba este periódico), mostrándose más activo durante los periodos en los cuales los moderados estuvieron vinculados al poder.

En relación con las vías de distribución debemos recordar que para aquéllos que no eran suscriptores y querían adquirir algunas de las piezas, deberían dirigirse a los mismos establecimientos en los que se suscribiría la Biblioteca de Cappa, destacando los almacenes de música y librerías extranjeras de Monier y Baillieri ${ }^{31}$.

Por otra parte, debemos tener en cuenta que la realización de esta empresa editorial hubiera sido prácticamente inviable sin la implicación de su director, dado el difícil momento político que atravesaba el país; es decir, Espín adoptó la figura de editor propia de la primera mitad del siglo XX (tal y como ya había sucedido años atrás con La Iberia Musical y Literaria), un hecho tan obvio que en la prensa se llegó a indicar que "la primera y segunda parte de la Biblioteca Musical Económica ha quedado terminada, merced al desprendimiento y laboriosidad del compositor Joaquín Espín y Guillén”32.

\footnotetext{
30 “Biblioteca Musical del maestro Espín y Guillén”, El Correo de los Teatros, 16-IX-1852, n. 42.

31 "Biblioteca lírica antigua y moderna”, El Clamor Público, 16-VIII-1851, n. 2179.

32 “Revista musical del año 1854”, La España, 2-I-1855, n. 2071.
} 


\section{Tesoro de Música Religiosa}

\section{Características generales}

La colección de música religiosa que publicó Espín, se diferencia de la Lira Sacro Hispana de Eslava en bastantes aspectos, entre ellos, la finalidad de la publicación, que se presentaba como una herramienta para "maestros de capilla, organistas de catedrales, villas, lugares, conventos de religiosas, escuelas elementales, colegios de enseñanza privada, etc." ${ }^{33}$. Además, se establecía que las obras pertenecerían a los compositores de mayor renombre y que serían de ejecución sencilla. Mientras, la antología propuesta por Eslava estaba centrada en los músicos españoles de los siglos XVI al XIX y constaba de cuatro secciones, una por cada siglo que abarcó. Es posible que la diferencia de planteamiento entre ambas empresas editoriales facilitase la pervivencia -al menos inicial- del Tesoro de Música Religiosa frente a la monumental Lira Sacro Hispana, que siguió publicándose hasta 1860. Necesariamente, el modo de organizar las entregas fue también distinto: las de la Lira se realizaban una vez al mes, y constaban de "veinte páginas de música en partición, del tamaño común, en buen papel, grabado y estampado en la calcografía del Sr. Catalina" ${ }^{34}$; el precio era de 12 reales por entrega, ascendiendo a 14 reales en provincias. Los no suscriptores podían hacerse con las piezas por 18 reales. Sin embargo, en el Tesoro se producían dos entregas mensuales, en las que se alternaban las dos secciones de la que constaba esta colección de música: una dedicada al órgano solo y otra a los cantos sagrados (con acompañamiento de órgano). Cada entrega estaba formada por "ocho láminas en cuarto mayor prolongado, impresas en riquísimo papel y su correspondiente cubierta” ${ }^{\text {"3 }}$. El precio estipulado era de 4 reales en Madrid y uno más en provincias, y el funcionamiento de la suscripción era el mismo que el estipulado para la Biblioteca Musical Económica. Del mismo modo, las 25 entregas constituían un tomo, cuya portada e índice también se repartían. Las piezas sueltas podían adquirirse al precio marcado en cada una de ellas (por ejemplo, el Tamtum Ergo de Rossini costaba 24 reales). La diferencia de precio entre la colección de música sacra de Espin y la monumental antología de Eslava es obvia y, en este caso, no es comparable, dada la distinta proyección y finalidad de ambas antologías.

\footnotetext{
33 "Sección de anuncios", La España, 12-IV-1853, n. 1543.

34 "Publicación de anuncios", La Esperanza, 21-VI-1853, n. 2655.

${ }^{35}$ Ibíd.
} 


\section{Repertorio}

En este caso, ha sido imposible reconstruir la totalidad de las entregas que configuraban el Tomo I de esta colección, tan sólo las 7 primeras entregas correspondientes a cada sección, lo que dificulta el estudio del repertorio. Se repartió el Tamtum Ergo de Rossini y una Misa fácil de Espín como parte de la sección dedicada a los cantos sagrados; en la dedicada al órgano solo, se entregó un Paso fugado de Parra, Tres divertimentos sobre el tema del Tamtum Ergo, de P. García, una Sonata-ofertorio de Espín y un Juego de versos perteneciente a R. P. Carrera. A pesar de que desconocemos el listado completo de los compositores que integran esta colección, parece claro que Espín la aprovechó también para dar a conocer sus propias obras.

\section{Edición, difusión y conservación del Tesoro de Música Religiosa}

Tan sólo hemos podido localizar la partitura de la Misa fácil de Espín, en su edición para el Tesoro de Música Religiosa. Las características de edición son similares a las comentadas para las partituras de la Biblioteca Musical Económica: tipografía de inspiración inglesa, portada sobria -acorde con la intención de una edición poco costosa- e introducción del precio en la primera lámina de la partitura (32 reales en este caso), la referencia a la colección a la que pertenece, y el tomo y número de entrega con el que se corresponde. Además, en la parte inferior derecha se introduce en todas las láminas de esta obra el número 2, que indica que ésta es la segunda pieza perteneciente a la sección de cantos sagrados; en la parte superior central se ha añadido el número de página del tomo.

En relación con la difusión, los anuncios correspondientes a esta empresa editorial privada se concentran nuevamente en el periódico La España, siendo puntual la aparición de anuncios en El Clamor Público, El Heraldo, La Época, y La Esperanza. No obstante, el volumen de anuncios localizados es mucho menor que en el caso de la Biblioteca Musical Económica, lo cual ha impedido constatar hasta qué momento se siguió publicando esta colección de música religiosa.

\section{Valoraciones finales}

Estas dos colecciones periódicas muestran un caso de transición entre la figura del editor musical propia de hasta mediados del siglo XIX, y el auténtico editor. Por una parte, tenemos la figura del compositor que arriesga su patrimonio para difundir sus obras, pero también la de otros compositores. Por otro lado, vemos que los almacenes de música ya 
funcionan como lugares de comercialización pero, sin embargo, Espín todavía asume la tramitación del proceso de edición calcográfica.

No fue ésta la primera incursión de Espín en el mundo editorial, pues anteriormente había invertido parte de su capital en el mantenimiento de La Iberia Musical y Literaria. Los suplementos repartidos con esta revista le valieron como experiencia previa para dirigir las dos colecciones musicales aquí estudiadas. El conocimiento de la demanda musical de los aficionados le llevó a establecer nuevamente, en el caso de la Biblioteca Musical Económica, dos secciones principales ya incluidas en los suplementos de su periódico. La diferencia radica en la elección del repertorio. En la sección de canto y piano, las canciones españolas pasan a tener un papel menos importante y sólo se publican algunos ejemplos de sus propias composiciones, alcanzando ahora las canciones italianas más importancia. Junto con sus obras, Espín incluyó las canciones de Rossini, dejando atrás las canciones de Dölher o Proch que formaron parte de los suplementos de La Iberia Musical y Literaria. En esta sección, destacan los fragmentos pertenecientes a la Gran Opéra, incluidos con aquéllos vinculados a la ópera italiana del primer y segundo romanticismo, lo que muestra un interés manifiesto por la ópera romántica francesa que tuvo una influencia visible en el género de la zarzuela. En la sección dedicada al piano solo se aprecia una tendencia similar a la sección anterior: desaparecen los ejemplos de compositores españoles -con excepción de las obras de Espín- y destaca Dölher, cuyas obras son ahora preferidas frente a las de Thalberg. La pequeña forma de salón sigue predominando, pero el vals ha cedido protagonismo a la polka y a la mazurka. Lo que no ha cambiado es el interés por las variaciones y fantasías sobre motivos de óperas conocidas, solo que ahora se aprecia una preferencia por el género de la Grand Opèra.

Al margen de la impresión de portadas que presentan grabados tipográficos sobrios, sin apenas motivos figurativos y sin ningún tipo de elemento extra que pudiese encarecer el precio de la obra, ésta era presentada en papel de buena calidad. Es muy probable que la reutilización de planchas haya sido un hecho, lo cual significaría un ahorro sustancial para el director de ambas colecciones musicales y permitiría descender su precio de comercialización. Una de las características editoriales de las obras pertenecientes a la Biblioteca Musical Económica y al Tesoro de Música Religiosa es la ausencia de un número de plancha como tal (entendiendo por ello la referencia al grabador y el número de 
serie de la plancha); de manera general, tan sólo se constata la colección a la que pertenece la obra (ya sea en su versión extendida o solamente mediante las siglas) y el número de entrega, una práctica ya utilizada por Espín en los suplementos de La Iberia Musical y Literaria. Desconocemos el volumen de las tiradas y el estudio de su distribución y difusión es harto difícil, puesto que la información proveniente de las fuentes hemerográficas es escasa, al igual que son limitadas las partituras conservadas.

Esta colección, al igual que los suplementos de La Iberia Musical y Literaria, estaba dirigida a un público eminentemente burgués, que además solía participar en las soirèes musicales características de mediados del siglo XIX. Pero también se debe recordar que la finalidad, tanto de la Biblioteca Musical Económica como del Tesoro de Música Religiosa, fue favorecer y dirigir la instrucción de profesores y diletantti en el primer caso, y de maestros de capilla, organistas y colegios privados, en el segundo. Esta intención educativa pone de manifiesto una atmósfera que se concretará a partir de 1854 en los ideales del kraussismo, y desde esta perspectiva deben entenderse estas dos iniciativas editoriales de Espín y su intención de que ambas fuesen asequibles económicamente, aspecto que se aprecia en el tipo de edición llevada a cabo para ambas colecciones de música. 